Homology, Homotopy and Applications, vol.12(1), 2010, pp.221-236

\title{
CATEGORIFIED SYMPLECTIC GEOMETRY AND THE STRING LIE 2-ALGEBRA
}

\author{
JOHN C. BAEZ AND CHRISTOPHER L. ROGERS
}

(communicated by J. Daniel Christensen)

\begin{abstract}
Multisymplectic geometry is a generalization of symplectic geometry suitable for $n$-dimensional field theories, in which the nondegenerate 2 -form of symplectic geometry is replaced by a nondegenerate $(n+1)$-form. The case $n=2$ is relevant to string theory: we call this '2-plectic geometry.' Just as the Poisson bracket makes the smooth functions on a symplectic manifold into a Lie algebra, the observables associated to a 2-plectic manifold form a 'Lie 2-algebra,' which is a categorified version of a Lie algebra. Any compact simple Lie group $G$ has a canonical 2-plectic structure, so it is natural to wonder what Lie 2-algebra this example yields. This Lie 2-algebra is infinite-dimensional, but we show here that the sub-Lie-2-algebra of left-invariant observables is finite-dimensional, and isomorphic to the already known 'string Lie 2-algebra' associated to $G$. So, categorified symplectic geometry gives a geometric construction of the string Lie 2-algebra.
\end{abstract}

\section{Introduction}

Symplectic geometry is part of a more general subject called multisymplectic geometry, invented by DeDonder [8 and Weyl [16] in the 1930s. In particular, just as the phase space of a classical point particle is a symplectic manifold, a classical string may be described using a finite-dimensional '2-plectic' manifold. Here the nondegenerate closed 2-form familiar from symplectic geometry is replaced by a nondegenerate closed 3-form.

Just as the smooth functions on a symplectic manifold form a Lie algebra under the Poisson bracket operation, any 2-plectic manifold gives rise to a 'Lie 2-algebra.' This is a categorified version of a Lie algebra: that is, a category equipped with a bracket operation obeying the usual Lie algebra laws up to isomorphism. Alternatively, we may think of a Lie 2-algebra as a 2-term chain complex equipped with a bracket satisfying the Lie algebra laws up to chain homotopy.

\footnotetext{
This work was partially supported by a grant from The Foundational Questions Institute. Received November 14, 2009, revised February 16, 2010; published on April 8, 2010. 2000 Mathematics Subject Classification: 70S05, 81T30, 53Z05, 53D05. Key words and phrases: categorification, string group, multisymplectic geometry. This article is available at http://intlpress.com/HHA/v12/n1/a12 Copyright (c) 2010, John C. Baez and Christopher L. Rogers. Permission to copy for private use granted.
} 
Now, every compact simple Lie group $G$ has a canonical 2-plectic structure, built from the Killing form and the Lie bracket. Which Lie 2-algebra does this example yield? Danny Stevenson suggested that the answer should be related to the already known 'string Lie 2-algebra' of $G$. Our main result here confirms his intuition. The Lie 2-algebra associated to the 2-plectic manifold $G$ comes equipped with an action of $G$ via left translations. The translation-invariant elements form a Lie 2-algebra in their own right, and this is the string Lie 2-algebra.

This gives a new geometric construction of the string Lie 2-algebra. For another construction, based on gerbes - or alternatively, central extensions of loop groups - see the paper by Baez, Crans, Schreiber and Stevenson 2. It will be interesting to see what can be learned from comparing these approaches.

The plan of the paper is as follows. In Section 2 we begin with a review of our recent paper [3] on 2-plectic geometry, Lie 2-algebras, and the classical bosonic string. The goal is to describe the Lie 2-algebra associated to a 2-plectic manifold. We only state theorems needed for the work at hand, referring the reader to the previous work for proofs and background material. More information on multisymplectic geometry can be found in papers by Cantrijn, Ibort, and de León [5] and by Cariñena, Crampin, and Ibort $\mathbf{7}$. Further details regarding the application of multisymplectic geometry to classical field theory can be found in the work of Kijowski [12, Gotay, Isenberg, Marsden, and Montgomery [9, Hélein [10, and Rovelli 14]. For Lie 2-algebras, see Baez and Crans [1] and also Roytenberg [15, whose approach we will follow in this paper.

In Section 3 we consider a Lie group acting on a 2-plectic manifold, preserving the 2-plectic structure. This group then acts on the associated Lie 2-algebra, and the invariant elements form a Lie sub-2-algebra. In Section 4 we apply this idea to the canonical 2-plectic structure on a compact Lie group $G$, where $G$ acts on itself by left translations. Finally, in Section $[5$ we show that the resulting Lie 2-algebra is none other than the string Lie 2-algebra.

\section{Acknowledgements}

We thank Danny Stevenson for suggesting that our construction of Lie 2-algebras from 2-plectic manifolds might yield the string Lie 2-algebra when applied to a compact simple Lie group. We also thank Dmitry Roytenberg for helpful conversations.

\section{2-plectic geometry and Lie 2-algebras}

We begin by defining 2-plectic manifolds and Lie 2-algebras. Then we explain how a 2-plectic manifold gives a Lie 2-algebra.

Definition 2.1. A 3 -form $\omega$ on a $C^{\infty}$ manifold $X$ is 2-plectic, or more specifically a 2-plectic structure, if it is both closed:

$$
d \omega=0,
$$

and nondegenerate:

$$
\forall v \in T_{x} X, \quad \iota_{v} \omega=0 \Rightarrow v=0
$$


where we use $\iota_{v} \omega$ to stand for the interior product $\omega(v, \cdot, \cdot)$. If $\omega$ is a 2-plectic form on $X$, then we call the pair $(X, \omega)$ a 2-plectic manifold.

Note that the 2-plectic structure induces an injective map from the space of vector fields on $X$ to the space of 2 -forms on $X$. This leads us to the following definition:

Definition 2.2. Let $(X, \omega)$ be a 2-plectic manifold. A 1-form $\alpha$ on $X$ is Hamiltonian if there exists a vector field $v_{\alpha}$ on $X$ such that

$$
d \alpha=-\iota_{v_{\alpha}} \omega
$$

We say $v_{\alpha}$ is the Hamiltonian vector field corresponding to $\alpha$. The set of Hamiltonian 1-forms and the set of Hamiltonian vector fields on a 2-plectic manifold are both vector spaces and are denoted as $\operatorname{Ham}(X)$ and $\operatorname{Vect}_{H}(X)$, respectively.

The Hamiltonian vector field $v_{\alpha}$ is unique if it exists, but note there may be 1 -forms $\alpha$ having no Hamiltonian vector field. Furthermore, two distinct Hamiltonian 1-forms may differ by a closed 1-form and therefore share the same Hamiltonian vector field.

We can generalize the Poisson bracket of functions in symplectic geometry by defining a bracket of Hamiltonian 1-forms. This can be done in two ways:

Definition 2.3. Given $\alpha, \beta \in \operatorname{Ham}(X)$, the hemi-bracket $\{\alpha, \beta\}_{\mathrm{h}}$ is the 1-form given by

$$
\{\alpha, \beta\}_{\mathrm{h}}=\mathcal{L}_{v_{\alpha}} \beta,
$$

where $\mathcal{L}_{v_{\alpha}}$ is the Lie derivative along the vector field $v_{\alpha}$.

Definition 2.4. Given $\alpha, \beta \in \operatorname{Ham}(X)$, the semi-bracket $\{\alpha, \beta\}_{\mathrm{s}}$ is the 1 -form given by

$$
\{\alpha, \beta\}_{\mathrm{s}}=\iota_{v_{\beta}} \iota_{v_{\alpha}} \omega
$$

These brackets in general will differ by an exact 1-form:

Proposition 2.5. Given $\alpha, \beta \in \operatorname{Ham}(X)$,

$$
\{\alpha, \beta\}_{\mathrm{h}}=\{\alpha, \beta\}_{\mathrm{s}}+d \iota_{v_{\alpha}} \beta .
$$

Proof. See Proposition 2 in $[\mathbf{3}$.

The space $\operatorname{Ham}(X)$ is closed under both brackets, but neither bracket satisfies all the axioms of a Lie algebra. The hemi-bracket fails to be skew-symmetric, while the semi-bracket fails to satisfy the Jacobi identity.

Proposition 2.6. Let $\alpha, \beta, \gamma \in \operatorname{Ham}(X)$ and let $v_{\alpha}, v_{\beta}, v_{\gamma}$ be the respective Hamiltonian vector fields. The hemi-bracket $\{\cdot, \cdot\}_{\mathrm{h}}$ has the following properties:

1. The bracket of Hamiltonian forms is Hamiltonian:

$$
d\{\alpha, \beta\}_{\mathrm{h}}=-\iota_{\left[v_{\alpha}, v_{\beta}\right]} \omega,
$$

so in particular we have

$$
v_{\{\alpha, \beta\}_{\mathrm{h}}}=\left[v_{\alpha}, v_{\beta}\right] .
$$


2. The bracket is skew-symmetric up to an exact 1-form:

$$
\{\alpha, \beta\}_{\mathrm{h}}+d S_{\alpha, \beta}=-\{\beta, \alpha\}_{\mathrm{h}}
$$

with $S_{\alpha, \beta}=-\left(\iota_{v_{\alpha}} \beta+\iota_{v_{\beta}} \alpha\right)$.

3. The bracket satisfies the Jacobi identity:

$$
\left\{\alpha,\{\beta, \gamma\}_{\mathrm{h}}\right\}_{\mathrm{h}}=\left\{\{\alpha, \beta\}_{\mathrm{h}}, \gamma\right\}_{\mathrm{h}}+\left\{\beta,\{\alpha, \gamma\}_{\mathrm{h}} \cdot\right\}_{\mathrm{h}}
$$

Proof. See Proposition 3 in [3].

Proposition 2.7. Let $\alpha, \beta, \gamma \in \operatorname{Ham}(X)$ and let $v_{\alpha}, v_{\beta}, v_{\gamma}$ be the respective Hamiltonian vector fields. The semi-bracket $\{\cdot, \cdot\}_{\mathrm{s}}$ has the following properties:

1. The bracket of Hamiltonian forms is Hamiltonian:

$$
d\{\alpha, \beta\}_{\mathrm{s}}=-\iota_{\left[v_{\alpha}, v_{\beta}\right]} \omega,
$$

so in particular we have

$$
v_{\{\alpha, \beta\}_{\mathrm{s}}}=\left[v_{\alpha}, v_{\beta}\right] .
$$

2. The bracket is antisymmetric:

$$
\{\alpha, \beta\}_{\mathrm{s}}=-\{\beta, \alpha\}_{\mathrm{s}} .
$$

3. The bracket satisfies the Jacobi identity up to an exact 1-form:

$$
\left\{\alpha,\{\beta, \gamma\}_{\mathrm{s}}\right\}_{\mathrm{s}}+d J_{\alpha, \beta, \gamma}=\left\{\{\alpha, \beta\}_{\mathrm{s}}, \gamma\right\}_{\mathrm{s}}+\left\{\beta,\{\alpha, \gamma\}_{\mathrm{s}}\right\}_{\mathrm{s}}
$$

with $J_{\alpha, \beta, \gamma}=-\iota_{v_{\alpha}} \iota_{v_{\beta}} \iota_{v_{\gamma}} \omega$.

Proof. See Proposition 4 in [3].

The observation that these brackets satisfy the Lie algebra laws 'up to exact 1-forms' leads to the notion of a Lie 2-algebra. Here we define a Lie 2-algebra to be a 2-term chain complex of vector spaces equipped with structures analogous to those of a Lie algebra, for which the usual laws hold up to coherent chain homotopy. Alternative definitions equivalent to the one given here are presented in [1] and [15.

Definition 2.8. A Lie 2-algebra is a 2-term chain complex of vector spaces $L=$ $\left(L_{0} \stackrel{d}{\leftarrow} L_{1}\right)$ equipped with the following structure:

- a chain map $[\cdot, \cdot]: L \otimes L \rightarrow L$ called the bracket,

- a chain homotopy $S: L \otimes L \rightarrow L$ from the chain map

$$
\begin{aligned}
L \otimes L & \longrightarrow L \\
x \otimes y & \longmapsto[x, y]
\end{aligned}
$$

to the chain map

$$
\begin{aligned}
L \otimes L & \longrightarrow L \\
x \otimes y & \longmapsto-[y, x]
\end{aligned}
$$

called the alternator, 
- a chain homotopy $J: L \otimes L \otimes L \rightarrow L$ from the chain map

$$
\begin{aligned}
& L \otimes L \otimes L \longrightarrow L \\
& x \otimes y \otimes z \longmapsto[x,[y, z]]
\end{aligned}
$$

to the chain map

$$
\begin{aligned}
L \otimes L \otimes L & \longrightarrow L \\
x \otimes y \otimes z & \longmapsto[[x, y], z]+[y,[x, z]]
\end{aligned}
$$

called the Jacobiator.

In addition, the following equations are required to hold:

$$
\begin{gathered}
{[x, J(y, z, w)]+J(x,[y, z], w)+J(x, z,[y, w])+[J(x, y, z), w]+[z, J(x, y, w)]} \\
=J(x, y,[z, w])+J([x, y], z, w)+[y, J(x, z, w)] \\
+J(y,[x, z], w)+J(y, z,[x, w]), \\
J(x, y, z)+J(y, x, z)=-[S(x, y), z] \\
J(x, y, z)+J(x, z, y)=[x, S(y, z)]-S([x, y], z)-S(y,[x, z]), \\
S(x,[y, z])=S([y, z], x) .
\end{gathered}
$$

Definition 2.9. A Lie 2-algebra for which the Jacobiator is the identity chain homotopy is called hemistrict. One for which the alternator is the identity chain homotopy is called semistrict.

Given a 2-plectic manifold $(X, \omega)$, we can construct both a hemistrict and a semistrict Lie 2-algebra. Both of these Lie 2-algebras have the same underlying 2-term complex, namely:

$$
L=\operatorname{Ham}(X) \stackrel{d}{\leftarrow} C^{\infty}(X) \stackrel{0}{\leftarrow} 0 \stackrel{0}{\leftarrow} 0 \stackrel{0}{\leftarrow} \cdots,
$$

where $d$ is the usual exterior derivative of functions. This chain complex is welldefined, since any exact form is Hamiltonian, with 0 as its Hamiltonian vector field.

The hemistrict Lie 2-algebra is equipped with a chain map called the hemibracket:

$$
\{\cdot, \cdot\}_{\mathrm{h}}: L \otimes L \rightarrow L
$$

In degree 0 , the hemi-bracket is given as in Definition 2.3 :

$$
\{\alpha, \beta\}_{\mathrm{h}}=\mathcal{L}_{v_{\alpha}} \beta .
$$

In degree 1 , it is given by:

$$
\{\alpha, f\}_{\mathrm{h}}=\mathcal{L}_{v_{\alpha}} f, \quad\{f, \alpha\}_{\mathrm{h}}=0 .
$$

In degree 2 , we necessarily have

$$
\{f, g\}_{\mathrm{h}}=0 .
$$

Here $\alpha, \beta \in \operatorname{Ham}(X)$, while $f, g \in C^{\infty}(X)$. 
Similarly, the semistrict Lie 2-algebra comes with a chain map called the semibracket:

$$
\{\cdot, \cdot\}_{\mathrm{s}}: L \otimes L \rightarrow L
$$

In degree 0 , the semi-bracket is given as in Definition 2.4:

$$
\{\alpha, \beta\}_{\mathrm{s}}=\iota_{v_{\beta}} \iota_{v_{\alpha}} \omega .
$$

In degrees 1 and 2, we set it equal to zero:

$$
\{\alpha, f\}_{\mathrm{s}}=0, \quad\{f, \alpha\}_{\mathrm{s}}=0, \quad\{f, g\}_{\mathrm{s}}=0 .
$$

The precise constructions of these Lie 2-algebras are given as follows:

Theorem 2.10. If $(X, \omega)$ is a 2-plectic manifold, then there is a hemistrict Lie 2-algebra $L(X, \omega)_{\mathrm{h}}$, where:

- the space of 0 -chains is $\operatorname{Ham}(X)$,

- the space of 1-chains is $C^{\infty}(X)$,

- the differential is the exterior derivative $d: C^{\infty}(X) \rightarrow \operatorname{Ham}(X)$,

- the bracket is $\{\cdot, \cdot\}_{\mathrm{h}}$,

- the alternator is the bilinear map $S: \operatorname{Ham}(X) \times \operatorname{Ham}(X) \rightarrow C^{\infty}(X)$ defined by $S_{\alpha, \beta}=-\left(\iota_{v_{\alpha}} \beta+\iota_{v_{\beta}} \alpha\right)$, and

- the Jacobiator is the identity chain homotopy, hence given by the trilinear map

$$
J: \operatorname{Ham}(X) \times \operatorname{Ham}(X) \times \operatorname{Ham}(X) \rightarrow C^{\infty}(X)
$$

with $J_{\alpha, \beta, \gamma}=0$.

Proof. See Theorem 5 in $\mathbf{3}$.

Theorem 2.11. If $(X, \omega)$ is a 2-plectic manifold, then there is a semistrict Lie 2-algebra $L(X, \omega)_{\mathrm{s}}$, where:

- the space of 0 -chains is $\operatorname{Ham}(X)$,

- the space of 1-chains is $C^{\infty}(X)$,

- the differential is the exterior derivative $d: C^{\infty}(X) \rightarrow \operatorname{Ham}(X)$,

- the bracket is $\{\cdot, \cdot\}_{\mathrm{s}}$,

- the alternator is the identity chain map, hence given by the bilinear map

$$
S: \operatorname{Ham}(X) \times \operatorname{Ham}(X) \rightarrow C^{\infty}(X)
$$

with $S_{\alpha, \beta}=0$, and

- the Jacobiator is the trilinear map

$$
J: \operatorname{Ham}(X) \times \operatorname{Ham}(X) \times \operatorname{Ham}(X) \rightarrow C^{\infty}(X)
$$

defined by $J_{\alpha, \beta, \gamma}=-\iota_{v_{\alpha}} \iota_{v_{\beta}} \iota_{v_{\gamma}} \omega$.

Proof. See Theorem 6 in $\mathbf{3}$.

A Lie 2-algebra homomorphism is a chain map between the underlying chain complexes that preserves the bracket up to 'coherent chain homotopy.' More precisely: 
Definition 2.12. Given Lie 2-algebras $L$ and $L^{\prime}$ with bracket, alternator and Jacobiator $[\cdot, \cdot], S, J$ and $[\cdot, \cdot]^{\prime}, S^{\prime}, J^{\prime}$ respectively, a homomorphism from $L$ to $L^{\prime}$ consists of:

- a chain map $\phi=\left(\phi_{0}, \phi_{1}\right): L \rightarrow L^{\prime}$, and

- a chain homotopy $\Phi: L \otimes L \rightarrow L^{\prime}$ from the chain map

$$
\begin{aligned}
L \otimes L & \longrightarrow L^{\prime} \\
x \otimes y & \longmapsto[\phi(x), \phi(y)]^{\prime}
\end{aligned}
$$

to the chain map

$$
\begin{aligned}
& L \otimes L \longrightarrow L^{\prime} \\
& x \otimes y \longmapsto \phi([x, y]),
\end{aligned}
$$

such that the following equations hold:

$$
\begin{gathered}
S^{\prime}\left(\phi_{0}(x), \phi_{0}(y)\right)-\phi_{1}(S(x, y))=\Phi(x, y)+\Phi(y, x), \\
J^{\prime}\left(\phi_{0}(x), \phi_{0}(y), \phi_{0}(z)\right)-\phi_{1}(J(x, y, z)) \\
=\left[\phi_{0}(x), \Phi(y, z)\right]^{\prime}-\left[\phi_{0}(y), \Phi(x, z)\right]^{\prime}-\left[\Phi(x, y), \phi_{0}(z)\right]^{\prime} \\
\quad-\Phi([x, y], z)-\Phi(y,[x, z])+\Phi(x,[y, z]) .
\end{gathered}
$$

The details involved in composing Lie 2-algebra homomorphisms are given by Roytenberg [15. We say a Lie 2-algebra homomorphism with an inverse is an isomorphism.

In fact, our previous work [3] shows that the hemistrict and semistrict Lie 2algebras associated to a 2-plectic manifold are isomorphic:

Theorem 2.13. There is a Lie 2-algebra isomorphism

$$
\phi: L(X, \omega)_{\mathrm{h}} \rightarrow L(X, \omega)_{\mathrm{s}}
$$

given by the identity chain map and a chain homotopy $\Phi: L \otimes L \rightarrow L$ that is nontrivial only in degree 0 , where it is given by

$$
\Phi(\alpha, \beta)=\iota_{v_{\alpha}} \beta
$$

for $\alpha, \beta \in L_{0}=\operatorname{Ham}(X)$.

Proof. See the proof of Theorem 7 in $\mathbf{3}$.

So, while the semistrict and hemistrict Lie 2-algebras defined above look different at first sight, we may legitimately speak of 'the' Lie 2-algebra associated to a 2-plectic manifold. We shall refer back to this result several times in the subsequent sections.

\section{Group actions on 2-plectic manifolds}

Next suppose we have a Lie group acting on a 2-plectic manifold, preserving the 2-plectic structure. In this situation both the hemistrict and semistrict Lie 2-algebras 
constructed above have Lie sub-2-algebras consisting of invariant Hamiltonian forms and functions.

More precisely, let $\mu: G \times X \rightarrow X$ be a left action of the Lie group $G$ on the 2-plectic manifold $(X, \omega)$, and assume this action preserves the 2-plectic structure:

$$
\mu_{g}^{*} \omega=\omega
$$

for all $g \in G$. Denote the subspace of invariant Hamiltonian 1-forms as follows:

$$
\operatorname{Ham}(X)^{G}=\left\{\alpha \in \operatorname{Ham}(X) \mid \forall g \in G \mu_{g}^{*} \alpha=\alpha\right\}
$$

The Hamiltonian vector field of an invariant Hamiltonian 1-form is itself invariant under the action of $G$ :

Proposition 3.1. If $\alpha \in \operatorname{Ham}(X)^{G}$ and $v_{\alpha}$ is the Hamiltonian vector field associated with $\alpha$, then $\mu_{g_{*}} v_{\alpha}=v_{\alpha}$ for all $g \in G$.

Proof. The exterior derivative commutes with the pullback of the group action. Therefore, if $v_{1}, v_{2}$ are smooth vector fields, then $d \alpha\left(\mu_{g_{*}} v_{1}, \mu_{g_{*}} v_{2}\right)=d \alpha\left(v_{1}, v_{2}\right)$, since we are assuming $\alpha$ is $G$-invariant. Since $\alpha \in \operatorname{Ham}(X)$, then $d \alpha=-\iota_{v_{\alpha}} \omega$, so

$$
\omega\left(v_{\alpha}, \mu_{g_{*}} v_{1}, \mu_{g_{*}} v_{2}\right)=\omega\left(v_{\alpha}, v_{1}, v_{2}\right)=\omega\left(\mu_{g_{*}} v_{\alpha}, \mu_{g_{*}} v_{1}, \mu_{g_{*}} v_{2}\right),
$$

where the last equality follows from $\mu_{g}{ }^{*} \omega=\omega$. Therefore,

$$
\omega\left(v_{\alpha}-\mu_{g_{*}} v_{\alpha}, \mu_{g_{*}} v_{1}, \mu_{g_{*}} v_{2}\right)=0 .
$$

Since $\omega$ is nondegenerate, and $v_{1}, v_{2}$ are arbitrary, it follows that $\mu_{g_{*}} v_{\alpha}=v_{\alpha}$.

Let $C^{\infty}(X)^{G}$ denote the subspace of invariant smooth functions on $X$ :

$$
C^{\infty}(X)^{G}=\left\{f \in C^{\infty}(X) \mid \forall g \in G \mu_{g}^{*} f=f \circ \mu_{g}=f\right\},
$$

and let $L^{G}$ denote the 2-term complex composed of $\operatorname{Ham}(X)^{G}$ and $C^{\infty}(X)^{G}$ :

$$
L^{G}=\operatorname{Ham}(X)^{G} \stackrel{d}{\longleftarrow} C^{\infty}(X)^{G}
$$

where $d$ is the exterior derivative.

The invariant differential forms on $X$ form a graded subalgebra that is stable under exterior derivative and interior product with an invariant vector field. Since the hemi-bracket and semi-bracket introduced in Definitions 2.3 and 2.4 are nothing but compositions of these operations, they restrict to well-defined chain maps:

$$
\begin{aligned}
& \{\cdot, \cdot\}_{\mathrm{h}}: L^{G} \otimes L^{G} \rightarrow L^{G}, \\
& \{\cdot, \cdot\}_{\mathrm{s}}: L^{G} \otimes L^{G} \rightarrow L^{G} .
\end{aligned}
$$

More precisely, we have the following proposition:

Proposition 3.2. If $\alpha, \beta \in \operatorname{Ham}(X)^{G}$ and $f \in C^{\infty}(X)^{G}$, then:

- $\{\alpha, f\}_{\mathrm{h}},\{f, \alpha\}_{\mathrm{h}} \in C^{\infty}(X)^{G}$,

- $\{\alpha, f\}_{\mathrm{s}},\{f, \alpha\}_{\mathrm{s}} \in C^{\infty}(X)^{G}$,

- $\{\alpha, \beta\}_{\mathrm{h}} \in \operatorname{Ham}(X)^{G}$,

- $\{\alpha, \beta\}_{\mathrm{s}} \in \operatorname{Ham}(X)^{G}$. 
Proof. By definition, $\{f, \alpha\}_{\mathrm{h}}=\{\alpha, f\}_{\mathrm{s}}=\{f, \alpha\}_{\mathrm{s}}=0$. Therefore they are trivially invariant under the group action. If $\alpha$ is an invariant Hamiltonian 1-form, then by Proposition 3.1 its Hamiltonian vector field $v_{\alpha}$ is invariant. Since $\{\alpha, \beta\}_{\mathrm{h}}=\mathcal{L}_{v_{\alpha}} \beta=$ $d \iota_{v_{\alpha}} \beta+\iota_{v_{\alpha}} d \beta$ and $\{\alpha, \beta\}_{\mathrm{s}}=\iota_{v_{\beta}} \iota_{v_{\alpha}} \omega$, it then follows from the above remarks that $\{\alpha, \beta\}_{\mathrm{h}}$ and $\{\alpha, \beta\}_{\mathrm{s}}$ are themselves invariant.

An important consequence of Proposition 3.2 is that $L^{G}$ forms a Lie sub-2-algebra of the hemistrict Lie 2-algebra $L(X, \omega)_{\mathrm{h}}$, which we call $L(X, \omega)_{\mathrm{h}}^{G}$. It also forms a Lie sub-2-algebra of $L(X, \omega)_{\mathrm{s}}$, which we call $L(X, \omega)_{\mathrm{s}}^{G}$. Furthermore, the isomorphism

$$
L(X, \omega)_{\mathrm{h}} \cong L(X, \omega)_{\mathrm{s}}
$$

of Theorem 2.13 restricts to an isomorphism between these Lie sub-2-algebras:

$$
L(X, \omega)_{\mathrm{h}}^{G} \cong L(X, \omega)_{\mathrm{s}}^{G} .
$$

We summarize these results in the next three corollaries:

Corollary 3.3. If $\mu: G \times X \rightarrow X$ is a left action of the Lie group $G$ on the 2-plectic manifold $(X, \omega)$ and for all $g \in G, \mu_{g}^{*} \omega=\omega$, then there is a hemistrict Lie 2-algebra $L(X, \omega)_{\mathrm{h}}^{G}$, where:

- the space of 0 -chains is $\operatorname{Ham}(X)^{G}$,

- the space of 1-chains is $C^{\infty}(X)^{G}$,

- the differential is the exterior derivative $d: C^{\infty}(X)^{G} \rightarrow \operatorname{Ham}(X)^{G}$,

- the bracket is $\{\cdot, \cdot\}_{\mathrm{h}}$,

- the alternator is the bilinear map $S: \operatorname{Ham}(X)^{G} \times \operatorname{Ham}(X)^{G} \rightarrow C^{\infty}(X)^{G}$ defined by $S_{\alpha, \beta}=-\left(\iota_{v_{\alpha}} \beta+\iota_{v_{\beta}} \alpha\right)$, and

- the Jacobiator is the identity, hence given by the trilinear map $J: \operatorname{Ham}(X)^{G}$ $\times \operatorname{Ham}(X)^{G} \times \operatorname{Ham}(X)^{G} \rightarrow C^{\infty}(X)^{G}$ with $J_{\alpha, \beta, \gamma}=0$.

Corollary 3.4. If $\mu: G \times X \rightarrow X$ is a left action of the Lie group $G$ on the 2-plectic manifold $(X, \omega)$ and for all $g \in G, \mu_{g}^{*} \omega=\omega$, then there is a semistrict Lie 2-algebra $L(X, \omega)_{\mathrm{s}}^{G}$, where:

- the space of 0 -chains is $\operatorname{Ham}(X)^{G}$,

- the space of 1-chains is $C^{\infty}(X)^{G}$,

- the differential is the exterior derivative $d: C^{\infty}(X)^{G} \rightarrow \operatorname{Ham}(X)^{G}$,

- the bracket is $\{\cdot, \cdot\}_{\mathrm{s}}$,

- the alternator is the identity, hence given by the bilinear map $S: \operatorname{Ham}(X)^{G}$ $\times \operatorname{Ham}(\mathrm{X})^{\mathrm{G}}(X)^{G} \rightarrow C^{\infty}(X)^{G}$ with $S_{\alpha, \beta}=0$, and

- the Jacobiator is the trilinear map $J: \operatorname{Ham}(X)^{G} \times \operatorname{Ham}(X)^{G} \times \operatorname{Ham}(X)^{G}$ $\rightarrow C^{\infty}(X)^{G}$ defined by $J_{\alpha, \beta, \gamma}=-\iota_{v_{\alpha}} \iota_{v_{\beta}} \iota_{v_{\gamma}} \omega$.

Corollary 3.5. The isomorphism

$$
\phi: L(X, \omega)_{\mathrm{h}} \rightarrow L(X, \omega)_{\mathrm{s}}
$$

restricts to a Lie 2-algebra isomorphism

$$
\phi: L(X, \omega)_{\mathrm{h}}^{G} \rightarrow L(X, \omega)_{\mathrm{s}}^{G} .
$$


Proof. As noted in Theorem 2.13, the isomorphism between $L(X, \omega)_{\mathrm{h}}$ and $L(X, \omega)_{\mathrm{s}}$ is given by the identity chain map and a chain homotopy $\Phi$ that is nontrivial only in degree 0:

$$
\Phi(\alpha, \beta)=\iota_{v_{\alpha}} \beta
$$

where $\alpha, \beta \in L_{0}=\operatorname{Ham}(X)$. If $\alpha$ and $\beta$ are invariant under the action of $G$, then it follows from Proposition 3.1 that $\iota_{v_{\alpha}} \beta$ is an invariant smooth function. Hence the chain homotopy $\Phi$ restricts to a chain homotopy $\Phi: L^{G} \otimes L^{G} \rightarrow L^{G}$.

\section{The 2-plectic structure on a compact simple Lie group}

Every compact simple Lie group has a canonical 2-plectic structure. This structure has been discussed in the multisymplectic geometry literature [5, 11, and it plays a crucial role in several branches of mathematics connected to string theory, including the theory of affine Lie algebras, central extensions of loop groups, gerbes, and Lie 2-groups [2, 4, 6, 13 .

Recall that if $G$ is a compact Lie group, then its Lie algebra $\mathfrak{g}$ admits an inner product $\langle\cdot, \cdot\rangle$ that is invariant under the adjoint representation Ad: $G \rightarrow \operatorname{Aut}(\mathfrak{g})$. For any nonzero real number $k$, we can define a trilinear form

$$
\theta_{k}(x, y, z)=k\langle x,[y, z]\rangle
$$

for any $x, y, z \in \mathfrak{g}$. Since the inner product is invariant under the adjoint representation, it follows that the linear transformations $\operatorname{ad}_{y}: \mathfrak{g} \rightarrow \mathfrak{g}$ given by $\operatorname{ad}_{y}(x)=[y, x]$ are skew adjoint. That is, $\left\langle\operatorname{ad}_{y}(x), z\right\rangle=-\left\langle x, \operatorname{ad}_{y}(z)\right\rangle$ for all $x, y, z \in \mathfrak{g}$. It follows that $\theta_{k}$ is totally antisymmetric. Moreover, $\theta_{k}$ is invariant under the adjoint representation since $\left[\operatorname{Ad}_{g}(x), \operatorname{Ad}_{g}(y)\right]=\operatorname{Ad}_{g}([x, y])$.

Let $L_{g}: G \rightarrow G$ and $R_{g}: G \rightarrow G$ denote left and right translation by $g$, respectively. Using left translation, we can extend $\theta_{k}$ to a left invariant 3-form $\nu_{k}$ on $G$. More precisely, given $g \in G$ and $v_{1}, v_{2}, v_{3} \in T_{g} G$ define a smooth section $\nu_{k}$ of $\Lambda^{3} T^{*} G$ by

$$
\left.\nu_{k}\right|_{g}\left(v_{1}, v_{2}, v_{3}\right)=\theta_{k}\left(L_{g^{-1} *} v_{1}, L_{g^{-1} *} v_{2}, L_{g^{-1} *} v_{3}\right) .
$$

It is straightforward to show that $\nu_{k}$ is also a right invariant 3 -form. Indeed, since $\operatorname{Ad}_{g}=L_{g *} \circ R_{g^{-1} *}$, the invariance of $\theta_{k}$ under the adjoint representation implies $R_{g}^{*} \nu_{k}=\nu_{k}$. From the left and right invariance we can conclude

$$
d \nu_{k}=0,
$$

since any $p$-form on a Lie group that is both left and right invariant is closed.

Now suppose that $G$ is a compact simple Lie group. Then $\mathfrak{g}$ is simple, so it has a canonical invariant inner product: the Killing form, normalized to taste. With this choice of inner product, the trilinear form $\theta_{k}$ is nondegenerate in the sense of Definition 2.1

Proposition 4.1. Let $G$ be a compact simple Lie group with Lie algebra $\mathfrak{g}$. If $x \in \mathfrak{g}$ and $\theta_{k}(x, y, z)=0$ for all $y, z \in \mathfrak{g}$, then $x=0$.

Proof. Recall that if $\mathfrak{g}$ is simple, then it is equal to its derived algebra $[\mathfrak{g}, \mathfrak{g}]$. Hence 
we may write any $x \in \mathfrak{g}$ as $x=\sum_{i=1}^{n}\left[y_{i}, z_{i}\right]$. Therefore,

$$
k\langle x, x\rangle=k \sum_{i=1}^{n}\left\langle x,\left[y_{i}, z_{i}\right]\right\rangle=\sum_{i=1}^{n} \theta_{k}\left(x, y_{i}, z_{i}\right)=0
$$

which implies $x=0$ since $\langle\cdot, \cdot\rangle$ is an inner product.

It is easy to see that the nondegeneracy of $\theta_{k}$ implies the nondegeneracy of $\nu_{k}$. Therefore, $\nu_{k}$ is a closed, nondegenerate 3 -form. We summarize the preceding discussion in the following proposition:

Proposition 4.2. Let $G$ be a compact simple Lie group with Lie algebra $\mathfrak{g}$. Let $\langle\cdot, \cdot\rangle$ be the Killing form, and let $k$ be a nonzero real number. The left-invariant 3-form $\nu_{k}$ on $G$ corresponding to $\theta_{k} \in \Lambda^{3} \mathfrak{g}^{*}$ is 2-plectic. So, $\left(G, \nu_{k}\right)$ is a 2-plectic manifold.

Now we wish to identify the Hamiltonian 1-forms associated with the 2-plectic structure $\nu_{k}$ that are invariant under left translation. We denote the space of all left invariant 1-forms as $\mathfrak{g}^{*}$. The left invariant Hamiltonian 1-forms, their corresponding Hamiltonian vector fields, and the left invariant smooth real-valued functions will be denoted as $\operatorname{Ham}(G)^{L}$, $\operatorname{Vect}_{H}(G)^{L}$, and $C^{\infty}(G)^{L}$, respectively.

If $f \in C^{\infty}(G)^{L}$, then by definition $f=f \circ L_{g}$ for all $g \in G$. Hence $f$ must be a constant function, so $C^{\infty}(G)^{L}$ may be identified with $\mathbb{R}$. The following theorem describes the left invariant Hamiltonian 1-forms:

Theorem 4.3. Every left invariant 1-form on $\left(G, \nu_{k}\right)$ is Hamiltonian. That is,

$$
\operatorname{Ham}(G)^{L}=\mathfrak{g}^{*} \text {. }
$$

Proof. Recall that if $\alpha$ is a smooth 1 -form and $v_{0}, v_{1}$ are smooth vector fields on any manifold, then

$$
d \alpha\left(v_{0}, v_{1}\right)=v_{0}\left(\alpha\left(v_{1}\right)\right)-v_{1}\left(\alpha\left(v_{0}\right)\right)-\alpha\left(\left[v_{0}, v_{1}\right]\right)
$$

Suppose now that $\alpha$ is a left invariant 1 -form on $G$ and $v_{0}, v_{1}$ are left invariant vector fields. Then the smooth functions $\alpha\left(v_{1}\right)$ and $\alpha\left(v_{0}\right)$ are also left invariant and therefore constant. Therefore the right-hand side of the above equality simplifies and we have

$$
d \alpha\left(v_{0}, v_{1}\right)=-\alpha\left(\left[v_{0}, v_{1}\right]\right) .
$$

Let $\alpha \in \mathfrak{g}^{*}$ and let $\langle\cdot, \cdot\rangle$ be the inner product on $\mathfrak{g}$ used in the construction of $\nu_{k}$. There exists a left invariant vector field $v_{\alpha} \in \mathfrak{g}$ such that $\alpha(x)=k\left\langle v_{\alpha}, x\right\rangle$ for all $x \in \mathfrak{g}$. Combining this with the above expression for $d \alpha$ gives

$$
d \alpha(x, y)=-k\left\langle v_{\alpha},[x, y]\right\rangle
$$

which implies

$$
d \alpha=-\iota_{v_{\alpha}} \nu_{k}
$$

Hence $\alpha \in \operatorname{Ham}(G)$, and $\operatorname{Ham}(G)^{L}=\operatorname{Ham}(G) \cap \mathfrak{g}^{*}=\mathfrak{g}^{*}$.

The most important application of Theorem 4.3 is that it allows us to use Corollaries 3.3 and 3.4 to construct hemistrict and semistrict Lie 2 -algebras both having $\mathfrak{g}^{*}$ as their space of 0 -chains, where $\mathfrak{g}$ is the Lie algebra of a compact simple Lie group. We summarize these facts in the following two corollaries: 
Corollary 4.4. If $G$ is a compact simple Lie group with Lie algebra $\mathfrak{g}$ and 2-plectic structure $\nu_{k}$, then there is a hemistrict Lie 2-algebra $L(G, k)_{\mathrm{h}}$, where:

- the space of 0 -chains is $\mathfrak{g}^{*}$,

- the space of 1-chains is $\mathbb{R}$,

- the differential is the exterior derivative $d: \mathbb{R} \rightarrow \mathfrak{g}^{*}$ (i.e., $d=0$ ),

- the bracket is $\{\cdot, \cdot\}_{\mathrm{h}}$,

- the alternator is the bilinear map $S: \mathfrak{g}^{*} \times \mathfrak{g}^{*} \rightarrow \mathbb{R}$ defined by

$$
S_{\alpha, \beta}=-\left(\iota_{v_{\alpha}} \beta+\iota_{v_{\beta}} \alpha\right),
$$

and

- the Jacobiator is the identity, hence given by the trilinear map

$$
J: \mathfrak{g}^{*} \times \mathfrak{g}^{*} \times \mathfrak{g}^{*} \rightarrow \mathbb{R}
$$

with $J_{\alpha, \beta, \gamma}=0$.

Corollary 4.5. If $G$ is a compact simple Lie group with Lie algebra $\mathfrak{g}$ and 2-plectic structure $\nu_{k}$, then there is a semistrict Lie 2-algebra $L(G, k)_{\mathrm{s}}$, where:

- the space of 0 -chains is $\mathfrak{g}^{*}$,

- the space of 1-chains is $\mathbb{R}$,

- the differential is the exterior derivative $d: \mathbb{R} \rightarrow \mathfrak{g}^{*}$ (i.e., $d=0$ ),

- the bracket is $\{\cdot, \cdot\}_{\mathrm{s}}$,

- the alternator is the identity, hence given by the bilinear map $S: \mathfrak{g}^{*} \times \mathfrak{g}^{*} \rightarrow \mathbb{R}$ with $S_{\alpha, \beta}=0$, and

- the Jacobiator is the trilinear map $J: \mathfrak{g}^{*} \times \mathfrak{g}^{*} \times \mathfrak{g}^{*} \rightarrow \mathbb{R}$ defined by

$$
J_{\alpha, \beta, \gamma}=-\iota_{v_{\alpha}} \iota_{v_{\beta}} \iota_{v_{\gamma}} \nu_{k} .
$$

Note that we obtain a hemistrict Lie 2-algebra $L(G, k)_{\mathrm{h}}$ and a semistrict Lie 2-algebra $L(G, k)_{\mathrm{s}}$ for every nonzero real number $k$. It is also important to recall that Corollary 3.5 implies the hemistrict Lie 2-algebra $L(G, k)_{\mathrm{h}}$ is isomorphic to the semistrict Lie 2-algebra $L(G, k)_{\mathrm{s}}$.

From the proof of Theorem 4.3, we see that there is a nice correspondence between left invariant Hamiltonian 1-forms and left invariant Hamiltonian vector fields, which relies on the isomorphism between $\mathfrak{g}$ and its dual space via the inner product $\langle\cdot, \cdot\rangle$. As a result, we have the following proposition, which will be useful in the next section:

Proposition 4.6. If $G$ is a compact simple Lie group with 2-plectic structure $\nu_{k}$ and $\langle\cdot, \cdot\rangle$ is the inner product on the Lie algebra $\mathfrak{g}$ of $G$ used in the construction of $\nu_{k}$, then $\operatorname{Vect}_{H}(G)^{L}=\mathfrak{g}$ and there is an isomorphism of vector spaces

$$
\varphi: \operatorname{Vect}_{H}(G)^{L} \stackrel{\sim}{\longrightarrow} \operatorname{Ham}(G)^{L}
$$

such that $\varphi(v)=k\langle v, \cdot\rangle$ is the unique left invariant Hamiltonian 1-form whose Hamiltonian vector field is $v$. 
Proof. We show only uniqueness since the rest of the proposition follows immediately from the arguments made in the proof of Theorem 4.3. Let $\alpha$ and $\beta$ be left invariant 1 -forms. The arguments made in the aforementioned proof imply $d \alpha=-\iota_{v_{\alpha}} \nu_{k}$ and $d \beta=-\iota_{v_{\beta}} \nu_{k}$, where $v_{\alpha}$ and $v_{\beta}$ are the unique left invariant vector fields such that $\alpha=k\left\langle v_{\alpha}, \cdot\right\rangle$ and $\beta=k\left\langle v_{\beta}, \cdot\right\rangle$. If $v_{\alpha}$ is the Hamiltonian vector field for both $\alpha$ and $\beta$ then $\iota_{v_{\alpha}} \nu_{k}=\iota_{v_{\beta}} \nu_{k}$. Hence by the nondegeneracy of the 2-plectic structure, $v_{\alpha}=v_{\beta}$ which implies $\alpha=\beta$.

\section{The string Lie 2-algebra}

We have described how to construct hemistrict and semistrict Lie 2-algebras from any compact simple Lie group $G$ and any nonzero real number $k$ using the 2-plectic structure $\nu_{k}$. Now we show that these are isomorphic to the 'string Lie 2-algebra' of $G$.

It was shown in previous work [1] that semistrict Lie 2-algebras can be classified up to equivalence by data consisting of:

- a Lie algebra $\mathfrak{g}$,

- a vector space $V$,

- a representation $\rho: \mathfrak{g} \rightarrow \operatorname{End}(V)$,

- an element $[j] \in H^{3}(\mathfrak{g}, V)$ of the Lie algebra cohomology of $\mathfrak{g}$.

A semistrict Lie 2-algebra $L$ is constructed from this data by setting the space of 0 -chains $L_{0}$ equal to $\mathfrak{g}$, the space 1 -chains $L_{1}$ equal to $V$, and the differential to be the zero map: $d=0$. The bracket $[\cdot, \cdot]: L \otimes L \rightarrow L$ is defined to be the Lie bracket on $\mathfrak{g}$ in degree 0 , and defined in degrees 1 and 2 by:

$$
[x, a]=\rho_{x}(a), \quad[a, x]=-\rho_{x}(a), \quad[a, b]=0,
$$

for all $x \in L_{0}$ and $a, b \in L_{1}$. The Jacobiator is taken to be any 3 -cocycle $j$ representing the cohomology class $[j]$.

From this classification we can construct the string Lie 2-algebra $\mathfrak{g}_{k}^{\mathrm{s}}$ of a compact simple Lie group $G$ by taking $\mathfrak{g}$ to be the Lie algebra of $G, V$ to be $\mathbb{R}, \rho$ to be the trivial representation, and

$$
j(x, y, z)=k\langle x,[y, z]\rangle,
$$

where $k \in \mathbb{R}$. When $k \neq 0$, the 3 -cocycle $j$ represents a nontrivial cohomology class. Note that since $\rho$ is trivial, the bracket of $\mathfrak{g}_{k}^{\mathrm{s}}$ is trivial in all degrees except 0 .

It is natural to expect that the string Lie 2-algebra is closely related to the Lie 2-algebra $L(G, k)_{\mathrm{s}}$ described in Corollary 4.5, since both are semistrict Lie 2-algebras built using solely the trilinear form $\theta_{k}$ on $\mathfrak{g}$. Indeed, this turns out to be the case:

Theorem 5.1. If $G$ is a compact simple Lie group with Lie algebra $\mathfrak{g}$ and 2-plectic structure $\nu_{k}$, then the string Lie 2-algebra $\mathfrak{g}_{k}^{\mathrm{s}}$ is isomorphic both to the semistrict Lie 2 -algebra $L(G, k)_{\mathrm{s}}$ and to the hemistrict Lie 2-algebra $L(G, k)_{\mathrm{h}}$.

Proof. Recall that in degree 0 , the bracket of $\mathfrak{g}_{k}^{\mathbf{s}}$ is the Lie bracket $[\cdot, \cdot]$ of $\mathfrak{g}$ and the bracket of $L(G, k)_{\mathrm{s}}$ is the bracket $\{\cdot, \cdot\}_{\mathrm{s}}$ introduced in Definition 2.4. In all other degrees, both brackets are the zero map. To simplify notation, $\mathfrak{g}_{k}^{\mathrm{s}}$ and $L(G, k)_{\mathrm{s}}$ will 
denote both the Lie 2-algebras and their underlying chain complexes. We will show that there exists a Lie 2-algebra isomorphism between $\mathfrak{g}_{k}^{\mathrm{s}}$ and $L(G, k)_{\mathrm{s}}$ by constructing a chain map

$$
\phi: \mathfrak{g}_{k}^{\mathrm{s}} \rightarrow L(G, k)_{\mathrm{s}}
$$

and a chain homotopy

$$
\Phi: \mathfrak{g}_{k}^{\mathrm{s}} \otimes \mathfrak{g}_{k}^{\mathrm{s}} \rightarrow L(G, k)_{\mathrm{s}},
$$

satisfying the conditions listed in Definition 2.12. Indeed, we will show that the maps $\{\cdot, \cdot\}_{\mathrm{S}} \circ(\phi \otimes \phi)$ and $\phi \circ[\cdot, \cdot]$ are actually equal.

By Proposition 4.6, there exists a vector space isomorphism

$$
\varphi: \operatorname{Vect}_{H}(G)^{L} \stackrel{\sim}{\longrightarrow} \operatorname{Ham}(G)^{L}
$$

which takes $x \in \operatorname{Vect}_{H}(G)^{L}$ to the left invariant Hamiltonian 1-form $\varphi(x)$ whose Hamiltonian vector field is $x$. The degree 0 components of $\mathfrak{g}_{k}^{\mathrm{s}}$ and $L(G, k)_{\mathrm{s}}$ are $\mathfrak{g}$ and $\mathfrak{g}^{*}$, respectively. From Proposition 4.6 and Theorem 4.3, we have

$$
\mathfrak{g}=\operatorname{Vect}_{H}(G)^{L}, \quad \mathfrak{g}^{*}=\operatorname{Ham}(G)^{L} .
$$

Using these equalities and the above isomorphism, we can define $\phi_{0}: \mathfrak{g} \rightarrow \mathfrak{g}^{*}$ to be the chain map $\phi$ in degree 0 with

$$
\phi_{0}(x)=\varphi(x) .
$$

The degree 1 component of both $\mathfrak{g}_{k}^{\mathrm{s}}$ and $L(G, k)_{\mathrm{S}}$ is $\mathbb{R}$, and so we define $\phi$ in degree 1 to be the identity map on $\mathbb{R}$.

If $x, y \in \mathfrak{g}$, then it follows from Proposition 4.6 that $\phi_{0}(x), \phi_{0}(y)$, and $\phi_{0}([x, y])$ are the unique left invariant Hamiltonian 1-forms whose Hamiltonian vector fields are $x$, $y$, and $[x, y]$, respectively. But Proposition 2.7 implies

$$
d\left\{\phi_{0}(x), \phi_{0}(y)\right\}_{\mathrm{s}}=-\iota_{[x, y]} \nu_{k} .
$$

Hence $[x, y]$ is also the Hamiltonian vector field of $\left\{\phi_{0}(x), \phi_{0}(y)\right\}_{\mathrm{s}}$. It then follows from uniqueness that $\left\{\phi_{0}(x), \phi_{0}(y)\right\}_{\mathrm{s}}=\phi_{0}([x, y])$. Therefore the chain maps

$$
\{\cdot, \cdot\}_{\mathrm{s}} \circ(\phi \otimes \phi): \mathfrak{g}_{k}^{\mathrm{s}} \otimes \mathfrak{g}_{k}^{\mathrm{s}} \rightarrow L(G, k)_{\mathrm{s}}
$$

and

$$
\phi \circ[\cdot, \cdot]: \mathfrak{g}_{k}^{\mathrm{s}} \otimes \mathfrak{g}_{k}^{\mathrm{s}} \rightarrow L(G, k)_{\mathrm{s}}
$$

are equal and hence the chain homotopy $\Phi$ can be taken to be the identity. It then follows that the equations in Definition 2.12 hold trivially, and the chain map $\phi$ is invertible by construction. Finally, by applying Corollary 3.5 , we see that $\mathfrak{g}_{k}^{\mathrm{s}}$ is also isomorphic to the hemistrict Lie 2-algebra $L(G, k)_{\mathrm{h}}$.

Roytenberg [15. has shown that given a simple Lie algebra $\mathfrak{g}$ and $k \in \mathbb{R}$, one can construct a hemistrict Lie 2-algebra $\mathfrak{g}_{k}^{\mathrm{h}}$, where:

- the space of 0 -chains is $\mathfrak{g}$,

- the space of 1-chains is $\mathbb{R}$,

- the differential $d$ is the zero map,

- the bracket is the Lie bracket of $\mathfrak{g}$ in degree 0 and trivial in all other degrees, 
- the alternator is the bilinear map $S(x, y)=-2 k\langle x, y\rangle$, and

- the Jacobiator is the identity.

He also showed that this hemistrict Lie 2-algebra $\mathfrak{g}_{k}^{\mathrm{h}}$ was isomorphic to the already known semistrict version of the string Lie 2 -algebra, which we are calling $\mathfrak{g}_{k}^{\mathrm{s}}$. Combining his result with Corollary 3.5 and Theorem 5.1, it becomes clear that we are dealing with the same Lie 2-algebra in four slightly different guises:

$$
\mathfrak{g}_{k}^{\mathrm{h}} \cong \mathfrak{g}_{k}^{\mathrm{s}} \cong L(G, k)_{\mathrm{h}} \cong L(G, k)_{\mathrm{s}} .
$$

In particular, we may view the Lie 2-algebras $L(G, k)_{\mathrm{h}}$ and $L(G, k)_{\mathrm{s}}$ as geometric constructions of $\mathfrak{g}_{k}^{\mathrm{h}}$ and $\mathfrak{g}_{k}^{\mathrm{s}}$, respectively.

\section{References}

[1] J.C. Baez and A.S. Crans, Higher-dimensional algebra VI. Lie 2-algebras, Theory Appl. Categ. 12 (2004), no. 15, 492-538. Also available as arXiv:math/ 0307263.

[2] J.C. Baez, D. Stevenson, A.S. Crans and U. Schreiber, From loop groups to 2-groups, Homology Homotopy Appl. 9 (2007), no. 2, 101-135. Also available as arXiv:math/0504123.

[3] J.C. Baez, A.E. Hoffnung and C.L. Rogers, Categorified symplectic geometry and the classical string, Comm. Math. Phys. 293 (2010), no. 3, 701-725. Also available as arXiv:0808.0246, with different theorem numberings.

[4] J.-L. Brylinski, Loop Spaces, Characteristic Classes and Geometric Quantization, Progr. Math. 107, Birkhäuser Boston, Inc., Boston, MA, 1993.

[5] F. Cantrijn, A. Ibort and M. de León, On the geometry of multisymplectic manifolds, J. Austral. Math. Soc. (Ser. A) 66 (1999), no. 3, 303-330.

[6] A.L. Carey, S. Johnson, M.K. Murray, D. Stevenson and B.-L. Wang, Bundle gerbes for Chern-Simons and Wess-Zumino-Witten theories, Comm. Math. Phys. 259 (2005), no. 3, 577-613. Also available as arXiv:math.DG/0410013.

[7] J.F. Cariñena, M. Crampin and L.A. Ibort, On the multisymplectic formalism for first order field theories, Differential Geom. Appl. 1 (1991), no. 4, 345-374.

[8] T. DeDonder, Theorie Invariantive du Calcul des Variations, Gauthier-Villars, Paris, 1935.

[9] M.J. Gotay, J. Isenberg, J.E. Marsden and R. Montgomery, Momentum maps and classical relativistic fields. Part I: covariant field theory, available as arXiv:physics/9801019.

[10] F. Hélein, Hamiltonian formalisms for multidimensional calculus of variations and perturbation theory, in Noncompact Problems at the Intersection of Geometry, Analysis and Topology (A. Bahri et. al., eds.), 127-147, Contemp. Math. 350, AMS, Providence, RI, 2004. Also available as arXiv:math-ph/0212036.

[11] A. Ibort, Multisymplectic geometry: generic and exceptional, in Proceedings of the IX Fall Workshop on Geometry and Physics (Vilanova i la Geltrú, 2000), 79-88 (X. Grácia et al., eds.), Publ. R. Soc. Math. Esp. 3, Real Sociedad Matemática Española, Madrid, 2001. 
[12] J. Kijowski, A finite-dimensional canonical formalism in the classical field theory, Comm. Math. Phys. 30 (1973), no. 2, 99-128.

[13] A. Pressley and G. Segal, Loop Groups, Oxford Math. Monogr., Oxford Univ. Press, New York, 1986.

[14] C. Rovelli, Covariant Hamiltonian formalism for field theory: Hamilton-Jacobi equation on the space $G$, available as arXiv:gr-qc/0207043

[15] D. Roytenberg, On weak Lie 2-algebras, in XXVI Workshop on Geometrical Methods in Physics, 80-198 (P. Kielanowski et al., eds.), AIP Conf. Proc. 956, Amer. Inst., Phys., Melville, NY, 2007. Also available as arXiv:0712.3461.

[16] H. Weyl, Geodesic fields in the calculus of variation for multiple integrals, Ann. of Math. 36 (1935), no. 3, 607-629.

John C. Baez baez@math.ucr.edu

Department of Mathematics, University of California, Riverside, CA 92521, USA

Christopher L. Rogers chris@math.ucr.edu

Department of Mathematics, University of California, Riverside, CA 92521, USA 